\title{
Tuberculose Multirresistente por Estirpes da Família Beijing, em Doentes de Lisboa, Portugal: Estudo Preliminar
}

\author{
Multidrug-Resistant Tuberculosis by Strains of Beijing \\ Family, in Patients from Lisbon, Portugal: Preliminary \\ Report
}

Fernando MALTEZ 1 , Teresa MARTINS ${ }^{1}$, Diana PÓVOAS ${ }^{1}$, João CABO ${ }^{1}$, Helena PERES ${ }^{2}$, Francisco ANTUNES $\rrbracket^{3}$, João PERDIGÃO ${ }^{4}$, Isabel PORTUGAL ${ }^{5}$

Acta Med Port 2017 Mar;30(3):175-184 - https://doi.org/10.20344/amp.7882

\section{RESUMO}

Introdução: As estirpes de Mycobacterium tuberculosis da família Beijing estão associadas à multirresistência. As estirpes da família Lisboa prevalecem entre os doentes com tuberculose multirresistente e extensivamente resistente desta região, mas vários estudos documentam a presença da família Beijing, embora desconhecendo-se as características dos doentes donde foram isoladas.

Material e Métodos: Estudo retrospectivo de 104 estirpes multirresistentes e extensivamente resistentes de Mycobacterium tuberculosis, isoladas e genotipadas, de 1993 a 2015, de igual número de doentes de Lisboa. Avaliámos a prevalência de ambas as famílias de estirpes e as características epidemiológicas e clínicas, dos infectados por estirpes Beijing.

Resultados: Setenta e quatro estirpes $(71,2 \%)$ pertenciam à família Lisboa, $25(24,0 \%)$ apresentavam padrão genotípico único e cinco $(4,8 \%)$ pertenciam à família Beijing, estas identificadas depois de 2009. Os infectados pela estirpe Beijing eram de nacionalidade angolana $(n=1)$, ucraniana $(n=2)$ e portuguesa $(n=2)$, na maioria jovens, quatro em cinco imunocompetentes e sem história de tuberculose anterior. Todos tinham tuberculose multirresistente. Não detectámos apresentações clínicas ou radiológicas diferenciadoras, nem padrão de resistências predominante. A taxa de cura foi alta (quatro doentes).

Discussão: Apesar do número de doentes infectados pela estirpe Beijing ser reduzido, sugere proporção importante de tuberculose primária, potencial de transmissão na comunidade, mas, também, melhor evolução clínica do que a descrita para outras estirpes, como a W-Beijing ou a Lisboa.

Conclusão: Apesar das estirpes da família Lisboa serem as principais responsáveis pelos casos de tuberculose multirresistente e extensivamente resistente na região de Lisboa, as estirpes Beijing transmitem-se na cidade e poderão modificar as características locais da epidemia.

Palavras-chave: Mycobacterium tuberculosis/genética; Portugal; Tuberculose Resistente a Múltiplos Medicamentos; Variação Genética

\section{ABSTRACT}

Introduction: Beijing family strains of Mycobacterium tuberculosis are associated with multidrug-resistance. Although strains of the Lisboa family are the most common among multidrug-resistant and extensively drug-resistant patients in the region, several studies have reported the presence of the Beijing family. However, the features of patients from whom they were isolated, are not yet known. Material and Methods: Retrospective study involving 104 multidrug-resistant and extensively drug-resistant strains of Mycobacterium tuberculosis, from the same number of patients, isolated and genotyped between 1993 and 2015 in Lisbon. We assessed the prevalence of strains of both families and the epidemiologic and clinical features of those infected with Beijing family strains.

Results: Seventy-four strains (71.2\%) belonged to the Lisboa family, $25(24.0 \%)$ showed a unique genotypic pattern and five (4.8\%) belonged to the Beijing family, the latter identified after 2009. Those infected with Beijing family strains were angolan $(n=1)$, ukrainian $(n=2)$ and portuguese $(n=2)$, mainly young-aged and, four of five immunocompetent and with no past history of tuberculosis. All had multidrug-resistant tuberculosis. We did not find any distinctive clinical or radiological features, neither a predominant resistance pattern. Cure rate was high (four patients)

Discussion: Although the number of infected patients with Beijing strains was small, it suggests an important proportion of primary tuberculosis, a potential for transmission in the community but also a better clinical outcome when compared to other reported strains, such as W-Beijing and Lisboa.

Conclusion: Although Lisboa family strains account for most of the multidrug and extensively drug-resistant tuberculosis cases in Lisbon area, Beijing strains are transmitted in the city and might change the local characteristics of the epidemics.

Keywords: Genetic Variation; Mycobacterium tuberculosis/genetics; Portugal; Tuberculosis, Multidrug-Resistant

\section{INTRODUÇÃO}

A tuberculose multirresistente (TB MR) e a tuberculose extensivamente resistente (TB ER) são um problema grave de Saúde Pública. ${ }^{1}$ No Mundo, em 2014, ocorreram 9,6 milhões de casos de tuberculose (TB). A tuberculose multir-

resistente representou $3,3 \%$ dos novos casos de TB e $20 \%$ dos casos previamente tratados, enquanto a TB ER representou $9,7 \%$ dos casos de tuberculose multirresistente. ${ }^{2}$ Em Portugal, em 2014, de acordo com a Direcção-Geral da

\footnotetext{
1. Serviço de Doenças Infecciosas. Hospital de Curry Cabral. Centro Hospitalar de Lisboa Central. Lisboa. Portugal.

2. Serviço de Patologia Clínica. Centro Hospitalar de Lisboa Central. Lisboa. Portugal.

3. Instituto de Saúde Ambiental. Faculdade de Medicina. Universidade de Lisboa. Lisboa. Portugal.

4. Instituto de Investigação do Medicamento (iMed.ULisboa). Faculdade de Farmácia. Universidade de Lisboa. Lisboa. Portugal.

5. Departamento de Microbiologia e Imunologia. Faculdade de Farmácia. Universidade de Lisboa. Lisboa. Portugal.

$\triangle$ Autor correspondente: Francisco Antunes. fantunes@medicina.ulisboa.pt

Recebido: 24 de maio de 2016 - Aceite: 28 de outubro de 2016 | Copyright @ Ordem dos Médicos 2017
} 
Saúde, a taxa de incidência de TB foi de 20,0/100 000 habitantes e a prevalência de TB MR, entre os casos notificados e testados no País, foi de $2,5 \%$, dos quais $26 \%$ representavam TB ER, especialmente concentrados na região de Lisboa e Vale do Tejo. Cerca de $78 \%$ destes casos de TB MR $(n=18)$ diziam respeito a doentes de nacionalidade portuguesa e os restantes $22 \%(n=5)$, a doentes oriundos da Europa de Leste $(n=1)$, da Europa Ocidental $(n=1)$, de um país Africano $(n=2)$ e de um país Asiático $(n=1){ }^{3}$

A nível global, a família de estirpes de Mycobacterium tuberculosis (M. tuberculosis) mais estudada é a Beijing. A primeira referência a esta família surgiu em 1995, quando foi identificada na província de Beijing (China) e na Mongólia. ${ }^{4}$ Depois, seria isolada em vários países vizinhos e, actualmente, constitui a família dominante na Ásia, com prevalências, por exemplo, de $48 \%$ no Vietname, de $44 \%$ na Tailândia, de $70 \%$ em Hong-Kong e excedendo $50 \%$ em muitos países, como na China e na Coreia do Sul. ${ }^{4-8}$ Num estudo efectuado numa província chinesa, $90 \%$ das estirpes eram Beijing e, destas, $27 \%$ eram multirresistentes (MR) e no Japão e em Taiwan representaram, respectivamente, $70 \%$ das estirpes extensivamente resistentes (ER) e $48 \%$ das estirpes MR. ${ }^{9-11}$ A família Beijing tem, também, grande prevalência na Rússia onde se documentou que era responsável por $48 \%$ dos casos de TB MR e por transmissão activa na comunidade. ${ }^{12-15} \mathrm{O}$ seu aparecimento nos Estados Unidos da América (EUA) foi, de igual modo, bem demonstrado. ${ }^{16,17} \mathrm{Em}$ Nova lorque, a estirpe $W$ da família Beijing emergiu no início dos anos 90 e foi responsável por grandes epidemias em hospitais e prisões, associadas à infecção por vírus da imunodeficiência humana (VIH), à multirresistência, a grande rapidez de transmissão e a tratamento complicado. ${ }^{16-18} \mathrm{Em}$ quatro hospitais daquela cidade afectou 267 doentes e causou uma mortalidade acima de $90 \%$, caracterizando-se pelo seu padrão de resistências e por alterações laboratoriais que lhe eram especificas. A análise genética das estirpes mostrava que $40 \%$ dos casos se relacionava com transmissão recente, contrariando a ideia de reactivação ou de toma incorrecta da medicação. ${ }^{16,19,20}$ Mais de 500 casos foram descritos em Nova lorque, entre 1990 e 1995, e espalhou-se a mais 10 estados norte-americanos, sendo responsável por mais de $25 \%$ dos casos de TB MR nos EUA. ${ }^{16,21}$

$\mathrm{Na}$ Europa, um estudo na Suécia mostrou que, apenas, $13 \%$ das estirpes MR eram Beijing e, a maioria destas, isolada de doentes imigrados da Ásia, não se documentando, apesar da proximidade geográfica com a Rússia e com os Países Bálticos, disseminação naquele País, na Dinamarca ou na Finlândia, que tinham incidências, igualmente, muito baixas. ${ }^{22} \mathrm{Na}$ Polónia, foi encontrada exclusivamente em $9 \%$ dos doentes com TB MR, sugerindo que tem responsabilidade na sua transmissão comunitária. ${ }^{23}$ Finalmente, em França, entre 2009 e 2010, foi encontrada predominância da família Beijing (75\%) entre as estirpes MR isoladas de doentes originários da Europa Central e de Leste e da Ásia, mas, também, identificada em doentes oriundos de França, contudo sem se ter estabelecido nexo epidemiológico entre os doentes. ${ }^{24}$

A família Beijing está, portanto, disseminada por todo o Mundo e aparece associada à multirresistência. No Irão, em 2009, foram identificados 15 doentes imunocompetentes infectados por estirpes de $M$. tuberculosis das famílias Beijing e Harlem, com resistência a todas as classes de antituberculosos de segunda linha, tendo sido designada por tuberculose totalmente resistente (TB TR). ${ }^{25} \mathrm{~A}$ distribuição mundial desta família é impressionante, continuando por esclarecer as suas características intrínsecas e os respectivos factores ambientais ou do hospedeiro que a definem. Embora já tenha estado envolvida (como a estirpe $\mathrm{N}$ ) em epidemias de tuberculose sensível e em clusters não associados à resistência verifica-se que, em muitas regiões (Estónia, Alemanha, África do Sul, Cuba), aparece associada à resistência aos antituberculosos e, geralmente, induzindo formas mais graves de TB, maior falência terapêutica e maior número de recaídas, do que outras famílias, continuando por entender, se tem maior facilidade em adquirir multirresistência ou se tem maior tendência para a transmissão. ${ }^{12,26-34}$ De igual modo, por caracterizar está a virulência desta família, definida pela capacidade em causar morbilidade e mortalidade excessivas.

Também em Portugal, nos anos de 1992 a 1994, era identificada, a partir das amostras de 124 doentes (infectados por VIH e imunocompetentes) de dois hospitais de Lisboa, uma família predominante, geneticamente distinta, designada por família Lisboa, por corresponder a um genotipo único, relacionado com o genotipo Latin-American-Mediterranean (LAM) ${ }^{35}$ Desde então, tem sido, ao longo dos anos, sucessivamente identificada, como a família prevalente entre os casos de TB MR e de TB ER, que ocorrem nesta região. ${ }^{36-39} \mathrm{Um}$ estudo de 2010, por exemplo, apontava para que mais de $50 \%$ das estirpes ER, a circular em Lisboa, pertencesse a esta família e, ainda, num outro estudo mais recente, demonstrou-se que a família Lisboa continua a ser dominante, propagando-se há mais de 20 anos. ${ }^{40,41} \mathrm{Em}$ paralelo, alguns estudos referem a identificação de estirpes da família Beijing, entre os casos de TB MR e de TB ER de Lisboa, com prevalências inferiores a 2,5\%, mas, até à data, nenhum caracterizou os doentes infectados por esta família, no que diz respeito à epidemiologia, à clínica e à evolução. ${ }^{42,43}$

\section{Objectivos}

Estudar as estirpes de M. tuberculosis isoladas e genotipadas de doentes com TB MR e TB ER de um Hospital de Lisboa, de 1993 a 2015. Avaliar a prevalência da família Lisboa e da família Beijing e, desta última, caracterizar a epidemiologia e a clínica, em particular os padrões de resistência aos antituberculosos e o prognóstico, em doentes com TB MR e TB ER da região de Lisboa.

\section{MATERIAL E MÉTODOS}

Realizámos um estudo retrospectivo, de todos os doentes com TB MR e TB ER do Serviço de Doenças Infecciosas do Hospital de Curry Cabral (HCC), em Lisboa, entre 1 
de Janeiro de 1993 e 31 de Dezembro de 2015 e, para os quais, estava disponível a caracterização genotípica e fenotípica das estirpes de $M$. tuberculosis isoladas em cultura. Identificámos as estirpes da família Lisboa e as estirpes da família Beijing.

Do processo clínico dos doentes infectados por estirpes Beijing, colhemos a informação demográfica, epidemiológica, clínica, laboratorial, microbiológica, terapêutica e evolutiva disponíveis. A TB foi classificada, de acordo com os critérios definidos pela OMS, em pulmonar e extrapulmonar. ${ }^{2}$ O diagnóstico de TB foi estabelecido pela identificação de bacilos ácido-álcool resistentes (BAAR) em exame directo (coloração de Ziehl-Neelsen) e por isolamento de $M$. tuberculosis em exame cultural, a partir de, pelo menos, uma amostra de material biológico. As amostras foram cultivadas em meio sólido de Lowenstein-Jensen, até 1995, e, a partir deste ano, também, em meio líquido de Bactec 460 TB (Becton Dickinson Diagnostic Instruments Systems ${ }^{\circledR}$, Towson, MD, USA), o qual foi substituído, em 2010, pelo meio líquido de Bactec MGIT 960 (Becton Dickinson Microbiology Systems $^{\circledR}$, Sparks, MD, USA). As estirpes de $M$. tuberculosis foram caracterizadas do ponto de vista fenotípico das resistências aos antituberculosos de primeira linha e aos antituberculosos de segunda linha, para as estirpes MR. Para os antibiogramas, segundo o método das proporções (1993 a 1994), utilizou-se o meio sólido de Middlebrook 7H11, de 1995 a 2010, o meio líquido de Bactec 460 TB e, a partir desta data, o meio líquido de Bactec MGIT 960. No meio sólido de Middlebrook 7H11 utilizaram-se os seguintes fármacos e respectivas concentrações - isoniazida (INH) - 0,2 $\mu \mathrm{g} / \mathrm{mL}$ e $0,5 \mu \mathrm{g} / \mathrm{mL}$, rifampicina (RMP) $-1,0$ $\mu \mathrm{g} / \mathrm{mL}$, estreptomicina (SM) - 2,0 $\mu \mathrm{g} / \mathrm{mL}$ ), etambutol (EMB) - 7,5 $\mu \mathrm{g} / \mathrm{mL}$, etionamida (ETO) - 10,0 $\mu \mathrm{g} / \mathrm{mL}$, cicloserina (CS) - $50 \mu \mathrm{g} / \mathrm{mL}$ e ácido para-aminosalicílico (PAS) - 0.5 $\mu \mathrm{g} / \mathrm{mL}$. No meio de Bactec 460 TB utilizaram-se INH $(0,1$ $\mu \mathrm{g} / \mathrm{mL})$, RMP $(2 \mu \mathrm{g} / \mathrm{mL})$, SM $(2 \mu \mathrm{g} / \mathrm{mL}$ e $6 \mu \mathrm{g} / \mathrm{mL})$, EMB $(2,5 \mu \mathrm{g} / \mathrm{mL}$ e $7,5 \mu \mathrm{g} / \mathrm{mL})$, pirazinamida (PZA) - $100 \mu \mathrm{g} / \mathrm{ml}$, capreomicina (CM) - 1,25 $\mathrm{gg} / \mathrm{mL}$ ), amicacina (AM) - 1,0 $\mu \mathrm{g} /$ $\mathrm{mL}$, canamicina $(\mathrm{KM})-5,0 \mu \mathrm{g} / \mathrm{mL}$, ofloxacina (OFX) - 2,0 $\mu \mathrm{g} / \mathrm{mL}$, esparfloxacina (SFX) - 0,4 $\mu \mathrm{g} / \mathrm{mL}$, rifabutina (RFB) - 0,5 $\mu \mathrm{g} / \mathrm{mL}$, ETO $(1,25 \mu \mathrm{g} / \mathrm{mL})$, clofazimina (CFZ) - 0,5 $\mu \mathrm{g}$ / $\mathrm{mL}$, PAS $(4,0 \mu \mathrm{g} / \mathrm{mL})$ e ciprofloxacina (CFX) - 0,5 $\mu \mathrm{g} / \mathrm{mL}$ e $1,0 \mu \mathrm{g} / \mathrm{mL}$. No meio de Bactec MGIT 960 utilizaram-se INH $(0,1 \mu \mathrm{g} / \mathrm{mL}), \operatorname{RMP}(1,0 \mu \mathrm{g} / \mathrm{mL}), \mathrm{SM}(1,0 \mu \mathrm{g} / \mathrm{mL})$, EMB $(5 \mu \mathrm{g} /$ $\mathrm{mL})$, PZA $(100 \mu \mathrm{g} / \mathrm{mL})$, ETO $(5,0 \mu \mathrm{g} / \mathrm{mL})$, OFX $(2,0 \mu \mathrm{g} / \mathrm{mL})$, CM $(1,25 \mu \mathrm{g} / \mathrm{mL}), A M(1,0 \mu \mathrm{g} / \mathrm{mL})$, linezolida (LZD) - 1,0 $\mu \mathrm{g} /$ $\mathrm{mL}$ e moxifloxacina (MFX) - 0,25 $\mu \mathrm{g} / \mathrm{mL}$. Definiu-se tuberculose multirresistente como aquela em que o agente foi resistente, pelo menos, à INH e à RMP e TB ER como tuberculose multirresistente, acrescida de resistência a qualquer fluoroquinolona e a qualquer antituberculoso injectável de segunda linha (CM, KM e AM). Definiu-se, ainda, TB TR, como aquela em que o agente mostrou resistência in vitro a todos os antituberculosos de primeira e segunda linhas testados. ${ }^{44}$ Resistência primária ou entre novos casos foi definida como a presença de resistência a um ou mais dos antituberculosos, em doentes nunca expostos a estes fár- macos. ${ }^{45}$ Resistência adquirida ou entre casos previamente tratados (ou secundária) foi definida como resistência a um ou mais dos antituberculosos, resultante de terapêutica insuficiente ou inapropriada, por má adesão por parte do doente, por má absorção dos fármacos ou por erro de prescrição. ${ }^{45}$

A tipificação das estirpes (identificação molecular) foi feita, até 2002, pelo método de restriction fragment length polymorphism, seguido de hibridização com a sequência de inserção 6110 (RFLP-IS6110) e, de 2003 em diante, pelo método de mycobacterial interspersed repetitive units-variable number of tandem repeats (MIRU-VNTR) e, finalmente, depois de 2009, também, por spacer oligonucleotide typing (Spoligotyping). ${ }^{46-48}$

Cluster foi definido como dois ou mais isolados, com padrões de RFLP-IS6110 diferindo em uma ou duas bandas ou como dois ou mais isolados com perfis semelhantes, pelo método MIRU-VNTR. Família foi definida como um grupo de isolados com homologia acima de $90 \%$. $^{35}$

Os doentes foram considerados como curados, quando, após terem completado o tratamento (mínimo de 18 ou 24 meses, após última cultura positiva), os sintomas desapareceram (cura clínica) e M. tuberculosis deixou de ser identificado na mesma amostra em que fora isolado previamente (cura microbiológica). A morte foi considerada relacionada com a TB, quando não se registou cura clínica e/ ou microbiológica no momento em que ocorreu. ${ }^{49}$

\section{RESULTADOS}

Incluímos 104 doentes residentes em Lisboa, dos quais se dispunha da caracterização genética das estirpes de M. tuberculosis MR ou ER. Não dispusemos, em nenhum doente, de mais do que uma genotipagem, nem de genotipagens provenientes de diferentes amostras.

Das 104 estirpes isoladas, 74 (71,2\%) constituíram dois clusters predominantes, já identificados nos anos 90 do século passado, sobretudo, em co-infectados por VIH, toxicodependentes e com má adesão ao tratamento do episódio de TB anterior (sem padrão de resistências aos antituberculosos), como pertencendo à família Lisboa. No período referente ao estudo, identificámos, ainda, cinco estirpes da família Beijing $(4,8 \%)$ e 25 estirpes $(24,0 \%)$ que, por apresentarem, individualmente, padrão genotípico único, foram designadas por 'outras'. Os polimorfismos eram muito variados entre as estirpes isoladas, prevalecendo as estirpes da família Lisboa, que foram sempre detectadas neste estudo, de 1993 a 2015. As estirpes da família Beijing, por seu lado, apenas foram identificadas a partir de 2009 (Tabela 1).

\section{Caracterização dos casos de infecção por estirpes da família Beijing}

No caso 1, a estirpe foi isolada da cultura de expectoração de doente do sexo masculino, caucasiano, de 56 anos, natural de Angola, temporariamente em Lisboa, imunocompetente, com história de TB pulmonar sensível aos antituberculosos (documentada dois anos antes, em 
Tabela 1 - Distribuição anual de 104 estirpes de M. tuberculosis multirresistente e extensivamente resistente

\begin{tabular}{lccc}
\hline \multirow{2}{*}{ Ano } & $\begin{array}{c}\text { Estirpe Lisboa } \\
\mathrm{n}\end{array}$ & $\begin{array}{c}\text { Estirpe Beijing } \\
\mathrm{n}\end{array}$ & $\begin{array}{c}\text { Outras Estirpes } \\
\mathrm{n}\end{array}$ \\
\hline 1993 & 5 & 0 & 1 \\
1994 & 1 & 0 & 0 \\
1995 & 17 & 0 & 4 \\
1996 & 18 & 0 & 4 \\
1997 & 1 & 0 & 0 \\
2000 & 1 & 0 & 0 \\
2001 & 5 & 0 & 0 \\
2003 & 4 & 0 & 1 \\
2004 & 7 & 0 & 3 \\
2005 & 4 & 0 & 1 \\
2006 & 0 & 0 & 1 \\
2007 & 1 & 0 & 1 \\
2009 & 0 & 1 & 1 \\
2010 & 1 & 1 & 2 \\
2011 & 2 & 0 & 3 \\
2012 & 3 & 0 & 0 \\
2013 & 0 & 1 & 2 \\
2014 & 1 & 0 & 1 \\
2015 & 3 & $(4,8)$ & 0 \\
\hline Total (\%) & $74(71,2)$ & & 0 \\
\hline & 1 & 0 & 0 \\
\hline
\end{tabular}

$\mathrm{n}$ : Número de estirpes ou doentes

Luanda), com má adesão ao tratamento e que se apresentou com TB MR, igualmente, de localização pulmonar. Os sintomas tiveram início 60 dias antes e o padrão imagiológico da radiografia e da tomografia computadorizada torácicas mostraram infiltrado intersticial difuso, nodular, bilateral, em toda a extensão de ambos os campos pulmonares, com cavitações, de igual modo, bilaterais, localizadas nos andares superiores e vértices. Os testes de sensibilidade aos antituberculosos de primeira e de segunda linha revelaram resistência à INH, RMP e ETO. A estirpe não foi identificada em nenhuma outra amostra biológica. $O$ doente foi tratado de acordo com o padrão de sensibilidades aos antituberculosos e considerado curado.

No caso 2, a estirpe foi identificada na expectoração de doente do sexo masculino, caucasiano, de 35 anos, natural da Ucrânia, residente em Lisboa desde há 10 anos, com co-infecção por VIH e por vírus da hepatite C (VHC), utilizador de drogas injectáveis (UDI), sem história clínica de tuberculose e de contactos com doentes com TB e a quem se diagnosticou TB miliar. Esta foi a infecção reveladora da imunodeficiência subjacente, com contagem de linfócitos $\mathrm{TCD}^{+}$de 62 células/ $/ \mathrm{L}$. A sintomatologia teve início 120 dias antes, sem nunca, até então, ter procurado assistência médica. Os exames imagiológicos pulmonares mostravam o padrão micronodular em 'grão de milho', micronódulos hepáticos e esplénicos e, ainda, tubérculos coroideus. A estirpe de $M$. tuberculosis foi identificada em amostras pulmonares e os testes de sensibilidade aos antituberculosos mostravam resistência à INH, RMP, EMB, SM, PZA, ETO, $\mathrm{KM}$ e AM. Apesar de medicado com antibacilares e antirretrovíricos, as culturas de expectoração não negativaram e faleceu, em resultado da TB, 86 dias após o início da terapêutica.

No caso 3, a estirpe foi isolada de doente do sexo feminino, caucasiana, de 38 anos, natural da Ucrânia, residente em Lisboa desde há 10 anos, imunocompetente e sem história clínica de TB anterior. Referiu contacto com irmão, falecido três anos antes na Ucrânia por TB MR e que a visitara em Lisboa. Trinta dias depois do início dos sintomas, foi-lhe diagnosticada TB pulmonar, com condensação alveolar e infiltrado nodular com cavitações do lobo superior e vértice do pulmão direito. Na expectoração foram identificados BAAR e o exame cultural com teste de sensibilidade aos antituberculosos mostrou $M$. tuberculosis resistente à INH, RMP, EMB, SM, PZA, ETO e AM. Iniciou terapêutica antituberculosa, que cumpriu com boa adesão, tendo, 29 dias depois, o exame directo da expectoração negativado e a cultura ao fim do segundo mês, com evolução para a cura.

No caso 4, a estirpe foi isolada de doente do sexo feminino, caucasiana, de 32 anos, natural de Lisboa, onde sempre residiu, imunocompetente e sem história de TB anterior. Cerca de 60 dias depois do início dos sintomas foi-Ihe diagnosticada TB pulmonar, com condensação e infiltrado nodular dos lobos médio e inferior do pulmão direito e, de igual modo, com cavitações. O diagnóstico de TB MR pulmonar foi fundamentado pelo padrão de resistência à

Tabela 2 - Características demográficas e epidemiológicas de cinco doentes com TB MR, infectados por estirpes de M. tuberculosis da família Beijing, entre 2009 e 2015

\begin{tabular}{|c|c|c|c|c|c|c|c|c|c|}
\hline Doente & Ano & Idade & Cor da pele & Sexo & Nacionalidade & Residência & VIH & $\begin{array}{c}\text { Tuberculose } \\
\text { anterior }\end{array}$ & $\begin{array}{c}\text { Contactos com doentes } \\
\text { de tuberculose }\end{array}$ \\
\hline 1 & 2009 & 56 & C & M & Angola & $\begin{array}{c}\text { Lisboa } \\
\text { (temporária) }\end{array}$ & Neg & $\operatorname{sim}$ & desconhecido \\
\hline 2 & 2010 & 35 & C & $\mathrm{M}$ & Ucrânia & $\begin{array}{l}\text { Lisboa } \\
\text { (10 anos })\end{array}$ & Pos & não & desconhecido \\
\hline 3 & 2013 & 38 & C & $\mathrm{F}$ & Ucrânia & $\begin{array}{l}\text { Lisboa } \\
\text { (10 anos) }\end{array}$ & $\mathrm{Neg}$ & não & $\operatorname{sim}$ \\
\hline 4 & 2014 & 32 & C & $\mathrm{F}$ & Portugal & Lisboa & $\mathrm{Neg}$ & não & $\operatorname{sim}$ \\
\hline 5 & 2014 & 36 & C & $\mathrm{M}$ & Portugal & Lisboa & Neg & não & $\operatorname{sim}$ \\
\hline
\end{tabular}

C: Caucasiano; F: feminino; M: Masculino; Neg: Negativo; Pos: Positivo; TB MR: Tuberculose multirresistente; VIH: Vírus da imunodeficiência humana 
INH, RMP, EMB, SM e ETO. Após o início da terapêutica, as culturas negativaram para $M$. tuberculosis, com evolução para a cura.

No caso 5, a estirpe foi isolada de um doente do sexo masculino, caucasiano, de 36 anos, residente em Lisboa e irmão da doente do caso anterior. Imunocompetente e sem história de TB no passado, com uma prova tuberculínica negativa, três meses antes. Vinte e um dias após o início de sintomas foi-lhe diagnosticada TB pulmonar, com infiltrado nodular e condensação com cavitações, nos lobos superiores e vértices de ambos os pulmões. A estirpe de M. tuberculosis mostrou um padrão de resistência aos antituberculosos de $1^{\mathrm{a}}$ e de $2^{\mathrm{a}}$ linha, sobreponível ao da irmã. Após o início da terapêutica, a expectoração revelou-se negativa aos 28 dias pelo exame microscópico e ao segundo mês pelo exame cultural, com evolução para a cura.

As cinco estirpes da família Beijing que identificámos neste estudo diziam respeito a um doente angolano, a dois ucranianos e a dois portugueses, todos a residir em Lisboa e, à excepção do caso 1 , todos eles tinham idades compreendidas entre os 32 e os 38 anos (Tabela 2). Apenas o doente do caso 1 , de nacionalidade angolana, tivera no seu País o diagnóstico prévio de TB sensível aos antibacilares, não tendo aderido ao tratamento e fazendo supor, em consequência, acumulação sequencial de resistências, isto é TB MR adquirida e importação da estirpe para Portugal. Os casos dos doentes ucranianos, residentes há 10 anos na região de Lisboa, sem história de TB no passado, faz supor TB MR primária. No caso 2, do doente co-infectado por $\mathrm{VIH}$, não eram conhecidos contactos suspeitos com outros casos de TB e não se podendo excluir a reactivação de estirpe de M. tuberculosis do País de origem, o facto de a TB MR se ter manifestado, apenas, em fase tão avançada de imunodepressão pode sugerir transmissão recente. Pelo contrário, a doente do caso 3 referia contacto recente com irmão falecido na Ucrânia, com TB MR, o qual terá introduzido a estirpe da família Beijing em Lisboa. Quanto aos casos 4 e 5 eram irmãos, conviventes na mesma habitação, sem história prévia de TB, sem saídas do País, e infectados por estirpes com teste fenotípico de resistências sobreponível, faz suspeitar, de igual modo, de TB MR primária e de transmissão recente. Aliás, o doente do caso 5 referia prova tuberculínica negativa, três meses antes do diagnóstico.

Para além das já descritas, não foram encontradas quaisquer ligações epidemiológicas entre os cinco doentes.

As manifestações clínicas e as alterações imagiológicas, identificadas em todos os doentes do estudo, são sobreponíveis às da TB clássica e nenhum dos aspectos foi considerado peculiar, não sendo de estranhar a disseminação tuberculosa e a ausência de cavitação pulmonar, no doente co-infectado por VIH e VHC (doente 2). À excepção deste doente, com TB miliar, todos os restantes, imunocompetentes, apresentavam TB pulmonar com cavitações e não tinham envolvimento extrapulmonar (Tabela 3). À excepção do doente co-infectado por $\mathrm{VIH}$, que só recorreu ao médico quatro meses depois do início do quadro clínico, 
todos os restantes procuraram os cuidados de saúde mais atempadamente.

O estudo microscópico da expectoração foi positivo, logo na primeira amostra dos doentes imunocompetentes, o que permitiu pôr em prática o teste molecular de resistências e o início imediato do tratamento para TB MR. Quanto ao segundo caso, o diagnóstico ocorreu em estado de doença avançada, com linfócitos TCD4 ${ }^{+}$de 62 células/ $\mu \mathrm{L}$ e o exame directo repetido da expectoração só foi positivo 12 dias após a admissão, o que teve como consequência o início tardio da terapêutica para TB MR. Nos cinco casos, as culturas confirmaram a multirresistência, não se tendo identificado nenhum caso de TB ER ou de TB TR (Tabela 4). Nos doentes imunocompetentes, a identificação de bacilos, por exame directo ou o isolamento da bactéria por cultura, ocorreu, exclusivamente, na expectoração, apesar de testadas outras amostras biológicas. Os padrões de resistência encontrados nas amostras dos cinco doentes eram diferentes de doente para doente, excepto para os doentes dos casos 4 e 5 . Todos os doentes imunocompetentes curaram.

\section{DISCUSSÃO}

Tal como já anteriormente demonstrado ${ }^{36,40,41}$ no presente estudo, a maioria de estirpes de $M$. tuberculosis em doentes com TB MR e TB ER da área de Lisboa pertence à família Lisboa ( $71,2 \%$ de 104 estirpes). Apenas 4,8\% (n =5) foram identificadas como pertencendo à família Beijing, apenas depois de 2009. Embora o objectivo do estudo não fosse o de avaliar a prevalência desta família, entre a população da região de Lisboa com TB MR e TB ER, é certo que a sua importância real na epidemia é maior, se tivermos em linha de conta a sua identificação nesta região, desde 1999, e as prevalências já referidas em algumas publicações ${ }^{41-43}$ e o facto da técnica de spoligotyping só ter sido utilizada no nosso estudo, de forma sustentada, a partir de 2009. O método de spoligotyping, introduzido em 1997, se bem que com menor capacidade discriminativa quando comparado com o RFLP-IS6110 e com a tipagem por MIRU-VNTR, é particularmente útil para a identificação das famílias Beijing, LAM, Haarlem, CAS e de outras estirpes e linhagens de $M$. tuberculosis, tendo emergido, como um método alternativo, rápido, sensível e custo-eficaz, que pode ser usado directamente na amostra, sem necessitar de cultura. ${ }^{48,50}$

A caracterização epidemiológica, clínica e evolutiva da amostra em estudo, constituída por um pequeno número de infectados pela por estirpes da família Beijing, mostrou que estas estirpes foram identificadas, principalmente, em doentes jovens, sem história de TB anterior, sem comorbilidades, imunocompetentes, sugerindo tratar-se, na maioria dos casos, de TB primária e de transmissão recente. Um estudo realizado no Vietname mostrou que $71 \%$ dos infectados por estirpes da família Beijing (54 de 76), tinha menos de 25 anos, concluindo que a disseminação da bactéria era, ali, recente. ${ }^{7}$ Outros estudos, com um número mais significativo de doentes, têm reconhecido algum protagonismo de estirpes desta família na transmissão activa na comunidade e na TB primária. ${ }^{10,11,15,23}$ Entretanto, na região de Lisboa desconhece-se o que possa ter acontecido com estas estirpes, nomeadamente, nos primeiros anos da epidemia de TB MR, em que predominavam os co-infectados por VIH, os UDI, a TB MR secundária à má adesão ao tratamento e a família Lisboa. ${ }^{36,38}$

Nos cinco doentes do estudo não identificámos quaisquer manifestações clínicas ou sinais radiológicos, que fossem atributos da TB causada por esta família Beijing quer nos doentes imunocompetentes, quer no co-infectado por $\mathrm{VIH}$, todos eles com TB pulmonar, contudo é de salientar a frequência das cavitações. Outros estudos, com maior número de doentes, não mostraram, também, qualquer relação da família Beijing com manifestações clínicas ou com alterações radiológicas específicas, incluindo a forma cavitada, identificando, apenas, a associação com resposta febril ao tratamento. ${ }^{51,52}$ Todos eles apresentaram TB pulmonar e a bactéria só foi identificada no exame directo e cultural das amostras biológicas colhidas na árvore respiratória (negativas no suco gástrico, urina e sangue dos imunocompetentes e, também, no líquor do co-infectado por VIH). Apenas o doente co-infectado por VIH tinha en-

Tabela 4 - Diagnóstico, padrões de resistência e evolução de cinco doentes com tuberculose multirresistente, infectados por estirpes de M. tuberculosis da família Beijing, entre 2009 e 2015

\begin{tabular}{|c|c|c|c|c|c|c|c|c|c|}
\hline Doente & $\begin{array}{l}\text { Diagnóstico } \\
\text { (dias) }\end{array}$ & Amostra & $\begin{array}{l}\text { Padrão de } \\
\text { resistências }\end{array}$ & INH & RMP & EMB & SM & PZA & ETO \\
\hline 1 & 1 & Expectoração & MR & $\mathrm{R}$ & $\mathrm{R}$ & $S$ & $S$ & $S$ & $\mathrm{R}$ \\
\hline 2 & 12 & $\begin{array}{l}\text { Expectoração, } \\
\text { secreções } \\
\text { brônquicas e LBA }\end{array}$ & MR & $\mathrm{R}$ & $\mathrm{R}$ & $\mathrm{R}$ & $\mathrm{R}$ & $\mathrm{R}$ & $\mathrm{R}$ \\
\hline 3 & 1 & Expectoração & MR & $\mathrm{R}$ & $\mathrm{R}$ & $\mathrm{R}$ & $\mathrm{R}$ & $\mathrm{R}$ & $\mathrm{R}$ \\
\hline 4 & 1 & Expectoração & MR & $\mathrm{R}$ & $\mathrm{R}$ & $\mathrm{R}$ & $\mathrm{R}$ & $S$ & $\mathrm{R}$ \\
\hline 5 & 1 & Expectoração & MR & $\mathrm{R}$ & $\mathrm{R}$ & $\mathrm{R}$ & $\mathrm{R}$ & $S$ & $\mathrm{R}$ \\
\hline
\end{tabular}

AM: Amicacina; CFX: Ciprofloxacina; CM: Capreomicina; CS: Cicloserina; EBM: Etambutol; ETO: Etionamida; INH: Isoniazida; KAN: Canamicina; LBA: Lavado broncoalveolar; LNZ: Linezolida; LVX: Levofloxacina; MOX: Moxifloxacina; NT: Não testado; OFX: Ofloxacina; PAS: Ácido para-aminosalicílico; PZA: Pirazinamida; R: Resistente; RMP: Rifampicina; S: Sensível; SM: Estreptomicina; MR: Multirresistente 
volvimento extrapulmonar concomitante, facilitado pela sua imunodepressão. A TB extrapulmonar, como se sabe, ocorre em 10 a $42 \%$ dos doentes com TB, dependendo da cor da pele ou etnia, da idade, da presença ou ausência de doença subjacente e do estado imunitário, mas também, do genotipo da estirpe de M. tuberculosis. ${ }^{53} \mathrm{~A}$ amostra do estudo é reduzida para se afirmar que a família Beijing se associa a um pequeno número de casos de TB extrapulmonar e se isso resulta de factores intrínsecos e de menor agressividade da bactéria ou do facto de afectar, maioritariamente, doentes imunocompetentes.

Nos doentes infectados pela família Beijing, o padrão de resistências diferiu e nenhum dos casos era de TB ER ou TB TR. Tal como mencionado noutros estudos, não se pode estabelecer uma correlação entre os padrões genéticos e os fenotipos de resistência aos antibacilares, dado que podem variar conforme o momento do isolamento e da realização do teste de sensibilidade, mas, no entretanto, não encontrámos um padrão predominante, não sendo possível afirmar, pelas dimensões da amostra, se estas estirpes têm vindo a acumular resistências ou a disseminarem-se na comunidade. ${ }^{36,38}$

Alguns trabalhos experimentais mostraram que a família Beijing, comparativamente a outras famílias, se associa a alterações patológicas mais graves no pulmão, com pneumonia e morte e, também, a resposta imunitária mais débil. ${ }^{54}$ Num estudo mais recente foi demonstrado que a família Beijing era um factor de risco de falência terapêutica e de recaída, independentemente da identificação (ou não) de resistências. ${ }^{55}$ Vários estudos têm mostrado, de igual forma, que esta estirpe está associada à indução de formas mais graves de TB e a maior número de casos de insucesso terapêutico, do que as estirpes de outras famílias. ${ }^{12,29,56}$ No nosso estudo, pelo contrário, encontrámos uma taxa alta de cura (quatro em cinco doentes). Todos os doentes imunocompetentes curaram, mas para esses bons resultados poderá ter contribuído o facto de o diagnóstico ter sido relativamente precoce, pela disponibilidade do teste molecular de resistências, o que permitiu o início rápido do tratamento. Após o início da terapêutica houve não só taxas altas de cura, mas também, rapidez na negativação do exame directo da expectoração, permanecendo os doentes por pouco tempo bacilíferos e com interrupção do risco de disseminação da infecção. O único doente falecido, infectado por VIH e em estádio de doença avançada, só recorreu aos cuidados de saúde, 120 dias após o início dos sintomas. As taxas de cura, se vierem a ser confirmadas num maior número de doentes, são muito diferentes, para melhor, do que as que se descreveram com outras estirpes de $M$. tuberculosis MR, nos anos 80 e 90, em outras regiões do Mundo, caracterizadas por elevada mortalidade e rapidez de progressão, como por exemplo as estirpes $W$ da família Beijing, nos EUA, ou as estirpes da família Lisboa, em Portugal. ${ }^{16,17,21,37,38,57}$

A família Beijing tem taxas de prevalência na Ásia, na Europa, na África e na América, respectivamente de 44,7\%, de $27,9 \%$, de $12,5 \%$ e de $8,9 \%$, estando $81,6 \%$ das estirpes associadas à resistência (com as maiores taxas a ocorrerem na China e na Rússia). ${ }^{4,5,58}$

Os métodos de genotipagem molecular têm um papel importante no entendimento da via de transmissão e de difusão mundial da TB MR e ER e permitem afirmar a existência de forte associação entre o País de origem do doente e a família de isolados MR. ${ }^{24}$ Das cinco estirpes da família Beijing identificadas, a primeira, em doente natural de Angola, foi importada deste País. A segunda e a terceira, em ucranianos, poderão ter sido importadas ou serem o resultado de transmissão recente em Lisboa, enquanto as duas restantes, em doentes portugueses, resultaram de transmissão na região. O facto da maioria dos doentes ser imunocompetente, jovens, sem história de TB anterior, sem factores de risco (alcoolismo ou sem abrigo, por exemplo) e sem comorbilidades pode ser um indiciador de potencial alto de transmissão na comunidade, das estirpes pertencentes a esta família.

Por outro lado, apesar de não haver ainda evidência da disseminação alargada de estirpes da família Beijing em Portugal (demonstrada por estudos epidemiológicos ou genotípicos), estes casos indicam que, regular ou irregularmente, estirpes provenientes de outras áreas geográficas invadem

\begin{tabular}{|c|c|c|c|c|c|c|c|c|c|c|c|}
\hline KAN & $\mathrm{CM}$ & AM & CFX & LVX & MOX & OFX & CS & PAS & LNZ & $\begin{array}{l}\text { Negativação } \\
\text { expectoração } \\
\text { (dias) }\end{array}$ & Evolução \\
\hline $\mathrm{R}$ & $S$ & $\mathrm{R}$ & NT & NT & NT & $S$ & $S$ & $S$ & NT & não & Falecido \\
\hline NT & $S$ & $\mathrm{R}$ & NT & NT & $S$ & $S$ & $S$ & $S$ & $S$ & 29 & Curado \\
\hline NT & $S$ & $S$ & NT & NT & $S$ & $S$ & $S$ & $S$ & $S$ & 30 & Curado \\
\hline NT & $S$ & S & NT & NT & $\mathrm{S}$ & $\mathrm{S}$ & S & S & S & 28 & Curado \\
\hline
\end{tabular}


o nosso ambiente, as quais poderão disseminar e tornar-se prevalentes e, inclusivé, agravar a evolução e o prognóstico da TB no País, o que é revelador de alguma instabilidade epidemiológica. Lisboa, lembre-se, é referência para a imigração de doentes provenientes de regiões onde a TB MR e as estirpes da família Beijing são prevalentes, como a África Subsariana ou a Europa de Leste.

\section{CONCLUSÃO}

A família Lisboa é a principal responsável pela epidemia de TB MR e de TB ER, que decorre na cidade, pelo menos desde 1993. Esta família foi prevalente nos doentes do nosso estudo ( $71,2 \%$ das estirpes genotipadas) e, de acordo com outros, poderá ter prevalências, entre os casos de TB MR e de TB ER de Lisboa, respectivamente de 47 a $61 \%$ e de 55 a $76 \% .^{59}$

A família Beijing representou apenas $4,8 \%$ das estirpes estudadas (cinco doentes, de 2009 a 2015), pelo que se comprova que há a introdução periódica, no nosso meio, de estirpes importadas de outras regiões geográficas. Embora, para já, com menor importância epidemiológica, estas estirpes espalham-se na comunidade e poderão vir a agravar ou a complicar o controlo da epidemia ou, mesmo, a tornar-se dominantes. Há evidência de que se transmitem na comunidade, embora não se possa afirmar, ainda, que estejam a acumular resistências. Em 2002, a família Beijing já representava, a nível global, $11 \%$ de todos os isolados testados e, considerando a ligação desta família à MR, a sua prevalência em certas regiões do Mundo e a globalização, ela poderá representar, se não controlada, um problema grave para o controlo da TB em Portugal. ${ }^{60}$

A grande limitação deste estudo é o pequeno número de estirpes da família Beijing identificadas, o que dificulta a sua caracterização epidemiológica e clínica. De qualquer forma, os resultados encontrados sugerem características particulares, que contrastam com a falência terapêutica descrita para esta e outras estirpes, em outras regiões geográficas, e que podem apontar para virulências diferentes, nomeadamente menor agressividade, menor letalidade, melhores taxas de cura e melhor prognóstico do que as es-

\section{REFERÊNCIAS}

1. Gandhi NR, Nunn P, Dheda K, Schaaf HS, Zignol M, van Soolingen D, et al. Multidrug-resistant and extensively drug-resistant tuberculosis: a threat to global control of tuberculosis. Lancet. 2010;375:1830-43.

2. World Health Organization. Global tuberculosis report 2015. Geneva: WHO; 2015.

3. Direcção-Geral da Saúde. Programa Nacional para a Infecção VIH/ SIDA. Portugal, Infecção VIH, SIDA e Tuberculose em números-2015. Lisboa: DGS; 2015.

4. van Soolingen D, Qian $L$, de Haas PE. Predominance of a single genotype of Mycobacterium tuberculosis in countries of east Asia. J Clin Microbiol. 1995;33:3234-8.

5. Glynn JR, Whiteley J, Bifani PJ, Kremer K, van Soolingen D. Worldwide ocurrence of Beijing/W strains of Mycobacterium tuberculosis: a systematic review. Emerg Infect Dis. 2002;8:843-9.

6. Anh DD, Borgdorff MW, Van LN, Lan NT, van Gorkom T, Kremer K, et al. Mycobacterium tuberculosis Beijing genotype emerging in Vietnam. Emerg Infect Dis. 2000;6:302-5.

7. Prodinger WM, Bunyaratvej P, Prachaktam R, Pavlic M. Mycobacterium tuberculosis isolates of Beijing genotype in Thailand. Emerg Infect Dis. 2001;7:483-4 tirpes W da família Beijing ou as estirpes da família Lisboa.

Este é o primeiro estudo a avaliar doentes da região de Lisboa, infectados por estirpes MR de M. tuberculosis, da família Beijing. A investigação molecular, conjugada com a epidemiologia e a clínica, deve ser expandida, de forma a melhorar a capacidade de detecção e controlo das cadeias de transmissão desta família e a optimizar a sensibilidade e precocidade do diagnóstico, contribuindo, assim, para a melhoria do seu prognóstico.

\section{AGRADECIMENTOS}

Os autores agradecem aos profissionais de saúde dos Serviços de Patologia Clínica e de Radiologia do Hospital de Curry Cabral-CHLC, aos do Laboratório de Micobactérias do Instituto Nacional de Saúde Dr. Ricardo Jorge e aos do Centro de Patogénese Molecular (Unidade dos Retrovírus e Infecções Associadas) da Faculdade de Farmácia da Universidade de Lisboa, pelo seu contributo na realização dos estudos complementares de diagnóstico, que tornaram possível a realização deste trabalho.

\section{PROTECÇÃO DE PESSOAS E ANIMAIS:}

Os autores declaram que os procedimentos seguidos estavam de acordo com os regulamentos estabelecidos pelos responsáveis da Comissão de Investigação Clínica e Ética e de acordo com a Declaração de Helsínquia da Associação Médica Mundial.

\section{CONFIDENCIALIDADE DOS DADOS:}

Os autores declaram ter seguido os protocolos do seu centro de trabalho acerca da publicação de dados.

\section{CONFLITOS DE INTERESSE:}

Os autores declaram não terem qualquer conflito de interesse relativamente ao presente artigo.

\section{FONTES DE FINANCIAMENTO:}

Os autores declaram não ter recebido subsídios ou bolsas para a elaboração do artigo.

8. Chan MY, Borgdorff M, Yip CW, de Haas PEW, Wong WS, Kam KM, et al. Seventy percent of the Mycobacterium tuberculosis isolates in HongKong represent the Beijing genotype. Epidemiol Infect. 2001;127:16971.

9. Wang J, Liu Y, Zhang CL, Ji BY, Zhang LZ, Shao YZ, et al. Genotypes and characteristics of clustering and drug susceptibility of Mycobacterium tuberculosis isolates collected in Heilongjiang Province, China. J Clin Microbiol. 2011;49:1354-62.

10. Murase Y, Maeda S, Yamada H, Okhado A, Chikamatsu K, Mizuno K, et al. Clonal expansion of multidrug-resistant and extensively drugresistant tuberculosis, Japan. Emerg Infect Dis. 2010;16:948-54.

11. Hsu AH, Lin CB, Lee YS, Chiang CY, Chen LK, Tsai YS, et al Molecular epidemiology of multidrug-resistant Mycobacterium tuberculosis in Eastern Taiwan. Int J Tuberc Lung Dis. 2010;14:924-6.

12. Toungoussa OS, Sandven P, Mariandsyshev AO, Nizovtseva NI, Bjune G, Caugant DA. Spread of the drug-resistant Mycobacterium tuberculosis strains of the Beijing genotype in the Archangel Oblast, Russia. J Clin Microbiol. 2002;40:1930-7.

13. Toungoussova O, Mariandyshev A, Bjune G, Sandven P, Caugant D. Molecular epidemiology and drug resistance of Mycobacterium 
tuberculosis isolates in the Archangel Prison in Russia: predominance of the W-Beijing clone family. Clin Infect Dis. 2003;37:665-72.

14. Drobniewski F, Balabanova Y, Nikolayevsky V, Ruddy M, Kuznetzov S, Zakharova S, et al. Drug-resistant TB, clinical virulence and the dominance of the Beijing strain family in Russia. JAMA. 2005;293:272631.

15. Afanas'ev MV, Ikryannikova LN, Il'ina EN, Kuz'min AV, Larionova EE, Smirnova TG, et al. Molecular typing of Mycobacterium tuberculosis circulated in Moscow, Russian Federation. Eur J Clin Microbiol Infect Dis. 2011;30:181-91

16. Bifani PJ, Plikaytis BB, Kapur V, Stockbauer K, Pan X, Lutfey ML, et al. Origin and interstate spread of a New York City multidrug-resistant Mycobacterium tuberculosis clone family. JAMA. 1996;275:452-7.

17. Hewlett D Jr, Franchini D, Horn D, Alfalla C, Yap R, Di Pietro D, et al. Outbreak of multidrug-resistant tuberculosis at a hospital - New York City, 1991. Morb Mortal Wkly Rep. 1993;42:427-33.

18. Frieden TR, Sterling T, Pablos-Mendez A, Kilburn JO, Cauthen GM, Dooley SW. The emergence of drug-resistant tuberculosis in New York City. N Engl J Med. 1993;328:521-6.

19. Alland D, Kalkut GE, Moss AR, McAdam RA, Hahn JA, Bosworth W, et al. Transmission of tuberculosis in New York City: an analysis by DNA fingerprinting and conventional epidemiological methods. N Engl J Med. 1994;330:1710-6.

20. Bifani P, Mathema B, Liu Z, Moghazeh SL, Shopsin B, Tempalski B, et al. Identification of a $\mathrm{W}$ variant outbreak of Mycobacterium tuberculosis via population-based molecular epidemiology. JAMA. 1999;282:2321-7.

21. Agerton TB, Valway SE, Blinkhorn RJ, Shilkret KL, Reves R, Schluter WW, et al. Spread of strain W, a highly drug-resistant strain of Mycobacterium tuberculosis, across the United States. Clin Infect Dis. 1999;29:85-92.

22. Ghebremichael S, Groenheit R, Pennhag A, Koivula T, Andersson $\mathrm{E}$, Bruchfeld $\mathrm{J}$, et al. Drug resistant Mycobacterium tuberculosis of the Beijing genotype does not spread in Sweden. PLoS ONE. 2010;5:e10893.

23. Jagielski T, Augstynowicz-Kopec E, Zozio T, Rastogi N, Zwolska $Z$. Spoligotype-based comparative population structure analysis of multidrug-resistant and isoniazid-monoresistant Mycobacterium tuberculosis complex clinical isolates in Poland. J Clin Microbiol. 2010;48:3899-909.

24. Sougakoff W. Molecular epidemiology of multidrug-resistant strains of Mycobacterium tuberculosis. Clin Microbiol Infect. 2011;17:800-5.

25. Velayati AA, Masjedi MR, Farnia P, Tabarsi P, Ghanavi J, Ziazarifi AH, et al. Emergence of new forms of totally drug-resistant tuberculosis bacilli: super extensively drug-resistant tuberculosis or totally drug-resistant strains in Iran. Chest. 2009;136:420-5

26. Milan SJ, Hauge KA, Kurepina NE, Lofy KH, Goldberg SV, Narita M, et al. Expanded geographical distribution of the $\mathrm{N}$ family of Mycobacterium tuberculosis strains within the United States. J Clin Microbiol. 2004;42:1064-8.

27. Caminero JA, Pena MJ, Campos-Herrero MI, Rodriguez JC, Garcia I, Cabrera P, et al. Epidemiological evidence of the spread of a Mycobacterium tuberculosis strain of the Beijing genotype on Gran Canaria island. Am J Respir Crit Care Med. 2001;164:1165-70.

28. Douglas JT, Qian L, Montoya JC, Musser JM, Van Embden JD, Van Soolingen D, et al. Characterization of the Manila family of Mycobacterium tuberculosis. J Clin Microbiol. 2003:41:2713-6.

29. Krüüner A, Hoffner SE, Sillastu H, Danilovits M, Levina K, Svenson SB, et al. Spread of drug-resistant pulmonary tuberculosis in Estonia. J Clin Microbiol. 2001;39:3339-45.

30. Niemann S, Rusch-Gerdes S, Richter E. IS6110 fingerprinting of drugresistant Mycobacterium tuberculosis strains isolated in Germany during 1995. J Clin Microbiol. 1997;35:3015-20

31. Streicher EM, Warren RM, Kewley C, Simpson J, Rastogi N, Sola C, et al. Genotypic and phenotypic characterisation of drug-resistant Mycobacterium tuberculosis isolates from rural districts of the Western Cape province of South Africa. J Clin Microbiol. 2004;42:891-4

32. Diaz R, Kremer K, de Haas PE, Gomez RI, Marrero A, Valdivia JA, et al. Molecular epidemiology of tuberculosis in Cuba outside of Havana, July 1994 - June 1995: utility of spoligotyping versus IS6110 restriction fragment length polymorphism. Int J Tuberc Lung Dis. 1998;2:743-50.

33. Velayati AA, Farnia P, Mirsaeidi M, Reza Masjedi M. The most prevalent Mycobacterium tuberculosis superfamilies among Iranian and Afghan TB cases. Scand J Infect Dis. 2006;38:463-8

34. Pfyffer GE, Strassle A, van Gorkum T, Portaels F, Rigouts L, Mathieu $\mathrm{C}$, et al. Multidrug-resistant tuberculosis in prison inmates, Azerbaijan. Emerg Infect Dis. 2001;7:855-61.
35. Portugal I. Epidemiologia molecular de estirpes de Mycobacterium tuberculosis isoladas em Lisboa: descrição preliminar de um genotipo único em Portugal. Rev Port Pneumol. 1996;2:393-401.

36. Portugal I, Brum L, Viveiros M, Moniz Pereira J, David H. Tipificação genética de estirpes multirresistentes de Mycobacterium tuberculosis isoladas na região de Lisboa. RPDI. 1998;21:54-9.

37. Hannan M, Peres H, Maltez F, Hayard AC, Machado J, Morgado R, et al. Investigation and control of a large outbreak of multi-drug resistant tuberculosis at a central Lisbon Hospital. J Hosp Infect. 2001;47:91-7.

38. Portugal I, Covas MJ, Brum L, Viveiros M, Ferrinho P, Moniz-Pereira J, et al. Outbreak of multiple drug-resistant tuberculosis in Lisbon: detection by restriction fragment length polymorphism analysis. Int J Tuberc Lung Dis. 1999;3:207-13.

39. Portugal I, Maia S, Moniz-Pereira J. Discrimination of multidrug-resistant Mycobacterium tuberculosis IS6110 fingerprinting subclusters by rpoB gene mutation analysis. J Clin Microbiol. 1999;37:3022-4.

40. Perdigão J, Macedo R, Malaquias A, Ferreira A, Brum L, Portugal I. Genetic analysis of extensively drug-resistant Mycobacterium tuberculosis strains in Lisbon, Portugal. J Antimicrob Chemother. 2010;65:224-7

41. Perdigão J, Silva H, Machado D, Macedo R, Maltez F, Silva C, et al. Unraveling Mycobacterium tuberculosis genomic diversity and evolution in Lisbon, Portugal, a highly drug resistant setting. BMC Genomics. 2014;15:991.

42. David S, Portugal C, Antunes A, Cardoso A, Calado A, Barros V, et al. Identificação molecular pelo método de Spoligotyping de estirpes do complexo Mycobacterium tuberculosis isoladas no Hospital Fernando Fonseca. Rev Port Pneumol. 2004;X:193-204.

43. David S, Barros V, Portugal C, Antunes A, Cardoso A, Calado A, et al. Novos dados sobre os Spoligotypes de estirpes do complexo Mycobacterium tuberculosis isoladas no Hospital Fernando Fonseca (Amadora-Sintra, Portugal). Rev Port Pneumol. 2005;XI:513-31.

44. Parida SK, Axelsson-Robertson R, Rao MV, Singh N, Master I, Lutckii $\mathrm{A}$, et al. Tottaly-drug resistant tuberculosis and adjunct therapies. J Int Med. 2015;277:388-405

45. Dye C, Scheele S, Dolin P, Pathania V, Raviglione MC. Consensus statement. Global burden of tuberculosis: estimated incidence, prevalence, and mortality by country. WHO Global Surveillance and Monitoring Project. JAMA. 1999;282:677-86

46. Van Soolingen D, Hermans PW, de Haas PE, Soll DR, van Embden JD. Occurrence and stability of insertion sequences in Mycobacterium tuberculosiscomplex strains: evaluation of an insertion sequencedependent DNA polymorphism as a tool in the epidemiology of tuberculosis. J Clin Microbiol. 1991;29:2578-86.

47. Supply P, Lesjean S, Savine E, Kremer K, van Soolingen D, Locht C Automated high-throughput genotyping for study of global epidemiology of Mycobacterium tuberculosis based on mycobacterial interspersed repetitive units. J Clin Microbiol. 2001;39:3563-71.

48. Kamerbeek J, Schouls L, Kolk A, van Agterveld M, Van Soolingen D, Kuijper S, et al. Simultaneous detection and strain differentiation of Mycobacterium tuberculosis for diagnosis and epidemiology. J Clin Microbiol. 1997; 35:907-14.

49. Mukherjee JS, Rich ML, Socci AR, Joseph JK, Viru FA, Shin SS, et al. Programmes and principles in treatment of multidrug-resistant tuberculosis. Lancet. 2004;363:474-81.

50. Brudey K, Driscoll J, Rigouts L, Prodinger WM, Gori A, Al-Hajoj SA, et al. Mycobacterium tuberculosis complex genetic diversity: mining the fourth international spoligotyping database (SpoLDB4) for classification, population genetics and epidemiology. BMC Microbiol. 2006;6:23.

51. van Crevel R, Nelwan $R H$, de Lenne W, Veeraragu $Y$, van der Zanden AG, Amin Z, et al. Mycobacterium tuberculosis Beijing genotype strains associated with febrile response to treatment. Emerg Infect Dis 2001;7:880-3.

52. Borgdorff MW, van Deutekom H, de Haas PE, Kremer K, van Soolingen D. Mycobacterium tuberculosis, Beijing genotype strains not associated with radiological presentattion of pulmonar tuberculosis. Tuberculosis. 2004:84:337-40

53. Caws M, Thwaites G, Dunstan S, Hawn TR, Lan NT, Thuong NT, et al. The influence of host and bacterial genotype on the development of disseminated disease with Mycobacterium tuberculosis. PloS Pathog. 2008;4:e1000034.

54. Lopez B, Aguilar D, Orozco H, Burger M, Espitia C, Ritacco V, et al. A marked difference in pathogenesis and immune response induced by different Mycobacterium tuberculosis genotypes. Clin Exp Immunol. 2003;133:30-7

55. Ngoc Lan NT, Lien HT, Tung LB, Borgdorff MW, Kremer K, van Soolingen 
D. Mycobacterium tuberculosis Beijing genotype and risk of treatment failure and relapse, Vietnam. Emerg Infect Dis. 2003;9:1633-5.

56. Cowley D, Govender D, February B, Wolfe M, Steyn L, Evans J, et al. Recent and rapid emergence of W-Beijing strains of Mycobacterium tuberculosis in Cape Town. S Afr Clin Infect Dis. 2008;47:1252-9.

57. Maltez F, Peres H, Machado J, Pina T, Cardoso O, Rodrigues P, et al. Multidrug resistant tuberculosis and HIV infection - a review concerning 152 patients. Varsóvia: $9^{\text {th }}$ European AIDS Conference; 2003.

58. Ramazanzadeh R, Sayhemiri K. Prevalence of Beijing family in
Mycobacterium tuberculosis in world population: systematic review and meta-analysis. Internat J Mycobact. 2014; 3:41-5.

59. Perdigão J, Macedo R, Silva C, Pinto C, Furtado C, Brum L, et al Tuberculosis drug-resistance in Lisbon, Portugal: a 6-year overview. Clin Microbiol Infect. 2011;17:1397-402.

60. Filliol I, Driscoll R, Rigouts L, Kreiswirth BN, Kremer K, Valetudie G. Global distribution of Mycobacterium tuberculosis spolygotypes. Emerg Infect Dis. 2002;8:1347-9. 